\title{
Research on Evaluation of Fire Detection Algorithms
}

\author{
JIAN $\mathrm{LI}^{1,2}$, WENHUI DONG ${ }^{1}$, ZHIBIN MEI ${ }^{1}$, and ZHUOFU WANG ${ }^{1}$ \\ ${ }^{1}$ Shenyang Fire Research Institute Ministry of Public Security \\ China, 110031 \\ ${ }^{2}$ Dalian University of Technology \\ China, 116023
}

\begin{abstract}
The main objective of this paper is to present an approach to evaluating algorithms of fire detection. The importance and purpose of evaluation of fire detection algorithms are briefly described. Characteristics of fire detection algorithms including fire detective, surroundings applicable and immunity to false alarm or nuisance sources are proposed as the main attributes using in evaluating process. Three kinds of evaluating patterns are studied and put into action, and then we called them physical pattern, interface pattern and simulation pattern. Detailed tests are designed to achieve scores under every pattern. Some examples, illustrating the application of this method to evaluate intelligent fire detection algorithms, are given.
\end{abstract}

KEYWORDS: detection, smoke, response patterns

\section{INTRODUCTION}

Fire detectors, which determine actual fire by responding fire resultant such as smoke, gases, flame or change of temperature, play important roles in fire automatic detection and alarm systems. The worth of a fire detector is determined as much by its ability not to respond to stimuli that are generated from non-threatening sources as to respond in a timely manner to an actual fire [1].

With the development of technology, a lot of research work has been done to test fire detectors performance. Cleary [2] use a test bed named as fire emulator detector evaluator (FE/DE) to examine fire detector's performance especially the ability that immune to stimuli not associated with a fire threat. Gockel [3] propose a modular model for fire sensors so as to know exactly how fire sensors'work. Lijian [4] proposed a new approach to evaluating fire detector's performance based on fuzzy analytic hierarchy process.

But how to evaluate a kind of fire detection algorithm especially intelligent algorithm is still a problem that we encountered. The reason related to the algorithm evaluation maybe associate with more and more fire detectors embedded complicated intelligent algorithms to distinguish fire signal and nuisance sources. So we can conclude that the fire detection algorithm evaluation is important and useful. By some well-chosen designed evaluating patterns and experiments, multiple aspects of an algorithms' performance can be demonstrated.

In order to point out fire detection algorithms' performance, multiple factors, which act mutually and co-adaptive in the whole evaluating process, must be distinction to insure the validity and scientific of the final result [4].

The remainder of this paper is organized as follows: Section 2 describes the main factors in the evaluating process. Section 3 describes the three evaluation patterns. Section 4 
contains the brief of experiments. Section 5 provides some evaluating examples to demonstrate this method. Section 6 contains a summary and conclusion.

\section{THE MAIN FACTORS IN THE EVALUATING PROCESS}

There are at least three factors that can influence the final evaluation result. First and foremost, fire detection algorithms are used to find out fire when it is at early stage. So, fire detective is the name we called to present the characteristic associated with fire detection algorithms' abilities to detect fire at early stage. Secondly, some buildings, especially in some representative location such as concourse, bibliotheca, shopping mall, have their own unique background signal that fire detectors explore. It is known that the sensor signal in clean air may change over the life of the detector. Such changes may be caused, for example, by contamination of the sensing chamber with dust or by other longterm effects such as component ageing [6]. We use surroundings applicable to indicate stability of detection algorithms working in different buildings with long-term effects. Thirdly owing to the performance when detectors are exploring in some nuisance circumstance such as smoking, cooking, bathing and so on, we call it immunity. So we take into account the three main factors when we discuss the problem of evaluating performance of fire detection algorithms.

\section{THE THREE EVALUATION PATTERNS}

The evaluation pattern one that we call it physical pattern is illustrated as Fig. 1. Needless to say, evaluation on the abilities of a kind of detection algorithm ties up with the whole detector itself, such as sensor, structure and circuit. Therefore, by the physical experiments, the performance of detector's algorithm can be demonstrated. Before we carrying out detailed test, Data that sampled from typical applicable surroundings must be gained. Fortunately, an elaborate project by Shenyang Fire research Institute, which funded by National government, has collected useful data , therefore a database has been built to support us as applicable information.

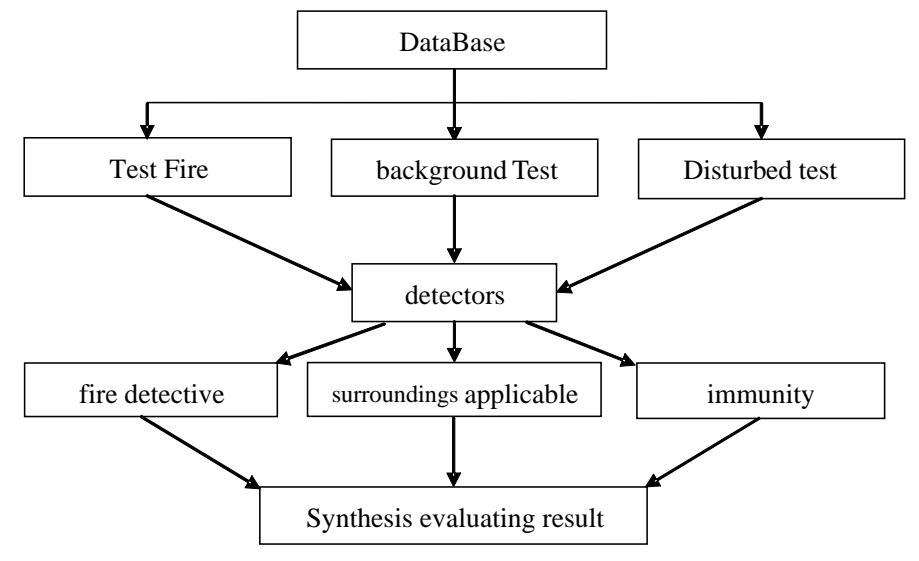

Fig. 1. Flow chart of the evaluation pattern one. 
Some tests have been designed to get each scores from the above three aspects. And every aspect includes a series tests. Finally, synthesis evaluating result is alluring to us, but how can we take order with so many attribute weights? Obviously, it should be formulated as a multiple criteria decision-making problem under uncertainty, detailed information can be reviewed in Lijian and Dongwenhui [4,5].

Figure 2 is the second evaluation pattern's flowchart. A set of test system is built up to carry out this kind of pattern. Firstly the channel between CPU A/D port and fire sensor's signal is cut off and connects to our test system's output port. Then start up application to put our simulated signal into detector's CPU A/D port, through response of the detector we can deduce the performance of its algorithm. Next, what kinds of signal can be used for our purpose, it also depends on the three aspects that we discuss in section 2, and before the evaluation process, signal from our database must be pre-processed such as uniform and so on.

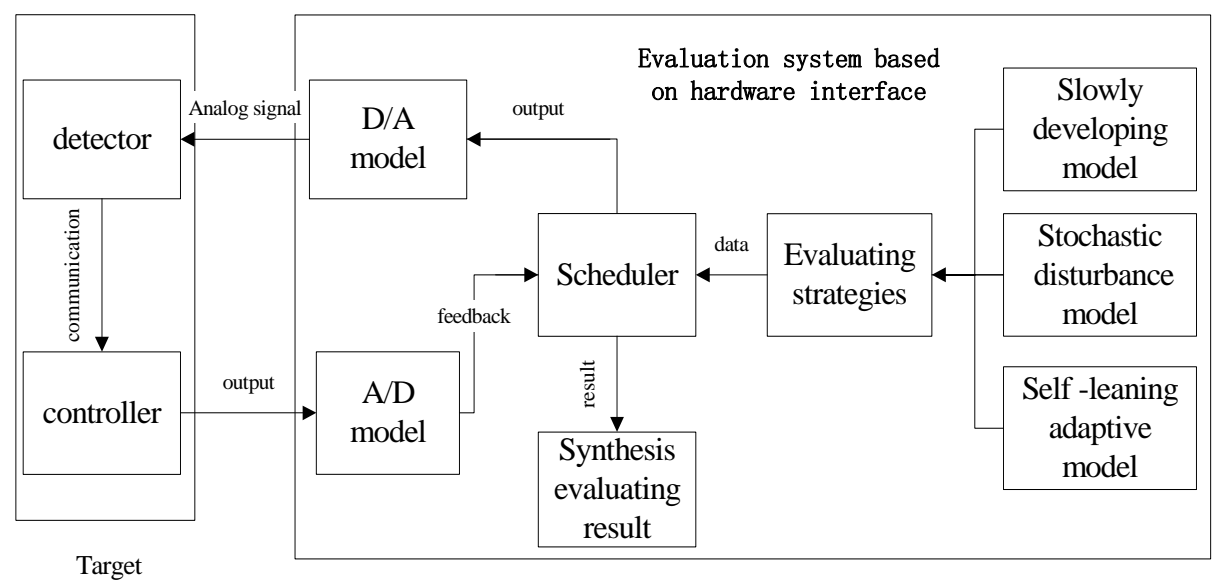

Fig. 2. Flow chart of the evaluation pattern two.

Considering of limits of physical experiments and requirements of academic research, the third evaluation pattern is proposed. Figure 3 is the flow chart. A standard program interface must be formulated firstly. The fire detection algorithm should be wrote in advance languages for example VC, VB (DLL, OCX) and so on, then by a set of evaluating strategies, the application uses data from the database as input to the algorithm and the response of the algorithm will be the result of whole evaluation process. 


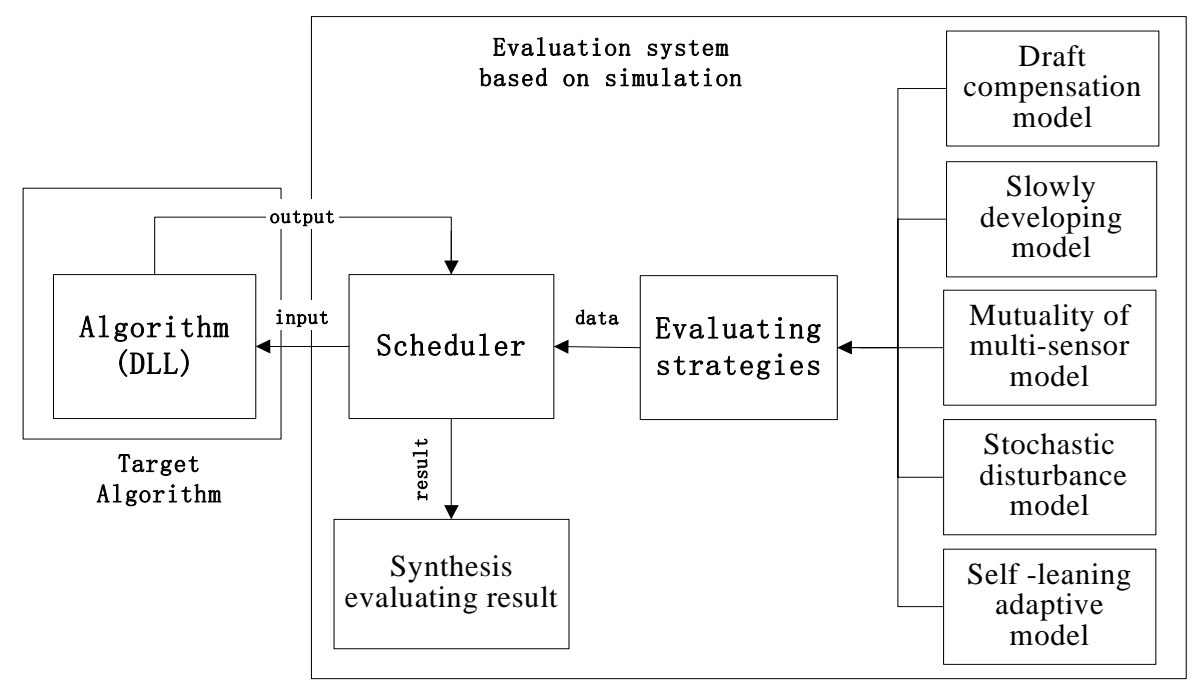

Fig. 3. Flow chart of the evaluation pattern.

\section{THE ITEMS OF EXPERIMENTS UNDER THE THREE EVALUATION PATTERN}

The experiments used in the evaluation process include two parts. On the one hand, standards including ISO/EN,UL and GB , especially fire test , are adopted. On the other hand, some experiments must be designed for certain purpose such as water mist, dust, and cooking or smoking. First and foremost, scores, which indicating the result of a experiment must be obtained through detailed design and try. Next, Adopting standards test insure those experiments more creditable and consistent.

Every evaluation pattern has its own suitable items of experiments. Some items are very difficult to accomplish in physical pattern whereas can be realized by simulation easily. Table 1 shows our designed items of experiments and corresponding methods.

Table 1. Items of experiments to three evaluation patterns.

\begin{tabular}{|c|c|c|c|}
\hline & Item & Standards & Pattern \\
\hline 1 & Slowly developing fire & $\begin{array}{c}\text { ISO_FDIS_7240- } \\
\text { 7/GB4715-2000 }\end{array}$ & 11 \\
\hline 2 & Compensation for draft & $\begin{array}{l}\text { ISO_FDIS_7240- } \\
\text { 7/GB4715-2000 }\end{array}$ & 1 \\
\hline 3 & Stochastic disturbance & / & 1 \\
\hline 4 & Self -leaning /adaptive & l & 1 \\
\hline 5 & Mist \dust\Cook\Smoke & / & \\
\hline 6 & Test fire( - ) & ISO/UL/EN54 & 11 \\
\hline
\end{tabular}




\section{EXAMPLES OF EXPERIMENTS}

\section{Slowly Developing Fire by Physical Pattern}

As is well known, ISO-7240-7 [6] has proposed the demands of slowly developing fire test but did not tell how to realize this test in detailed. We attempted to accomplish this task and came to terms that this test must take into account that the accumulation of smoke particles and diameter distribution then to satisfy the demands of standards in present because of the rates of change A/4 per hour is very slowly. Even if this test can be realized easily under pattern two and three, we also try to finish it with physical experiment through reduce fuel and increase test duration and so on. For a kind of detector $\mathrm{A}=0.12 \mathrm{db} / \mathrm{m}$, the actual result $\mathrm{db} / \mathrm{m}$-second is shown in Fig. 4 .

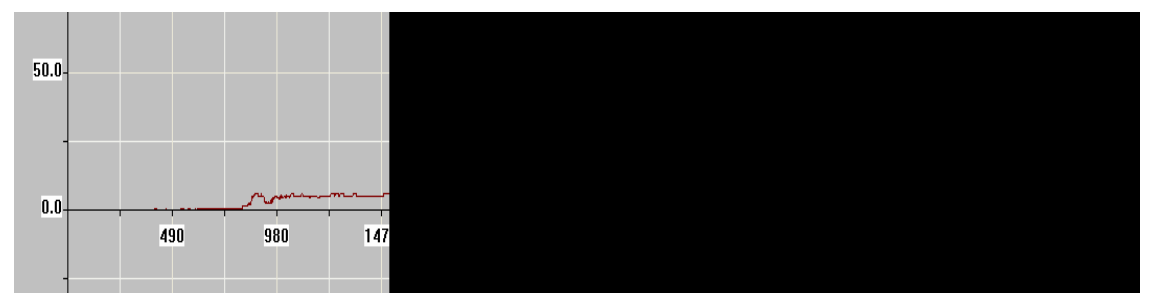

Fig. 4. The result of slowly developing fire.

\section{Test Fire by Simulation Pattern}

We have achieved a type of detector's actual fire detection algorithm in VC++ DLL format and take it as target to test our pattern and method. Through a series of items of test can we declare this pattern is useful and efficient, see Fig. 5.

\section{Stochastic Disturbance Test by Interface Pattern}

To test the stability of the target algorithm we utilized the method of routine signal coupling stochastic disturbance generated by software. Just like Rainer [7] used artificial signal events to test detection algorithms, the generated events have random nature, i.e., the signal shape, amplitude and duration is randomly varied and the points in time [7]. But in our research we focused on the routine monitoring signal of the sensor coupling random pulse. The performance of the detection algorithm can be gained through the detector actual output. Figure 6 shows this scenic of the experiment. 


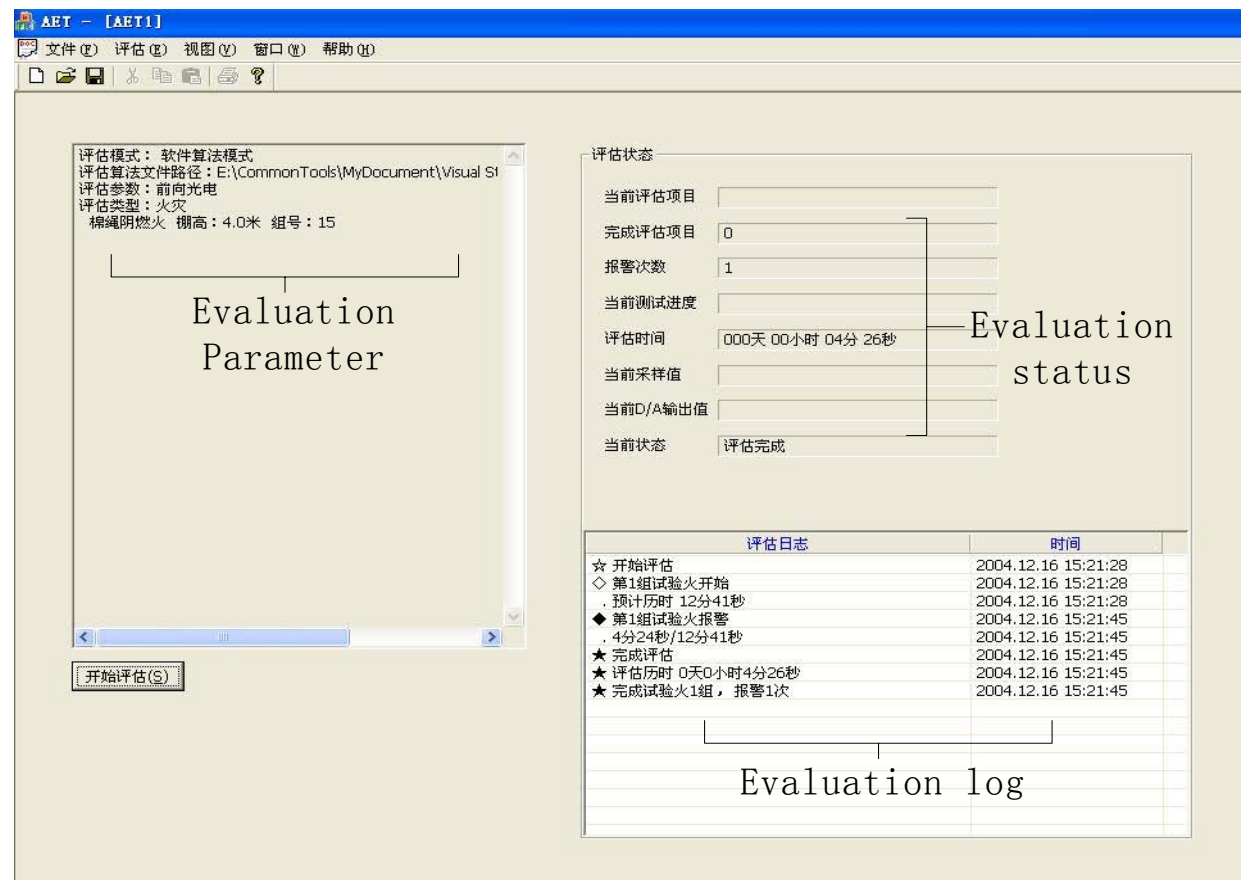

Fig. 5. The test of simulation pattern.

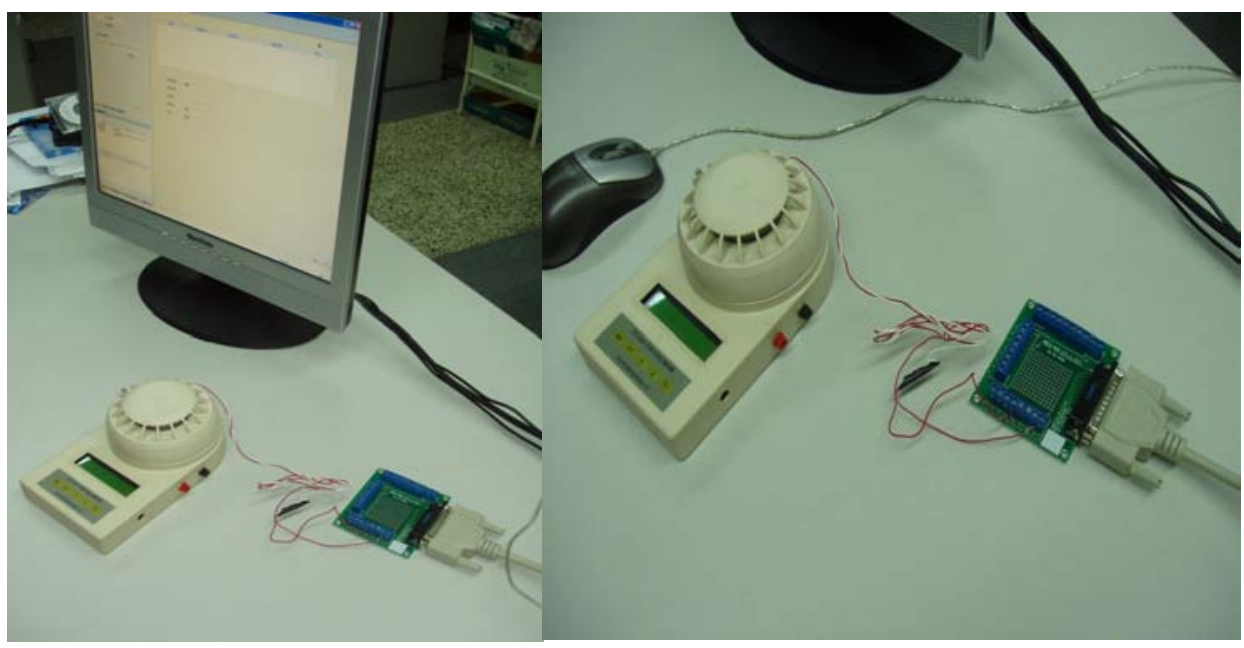

Fig. 6. The example of pattern II.

\section{SUMMARY AND CONCLUSIONS}

In this paper we have studied the process of evaluating performance of detection algorithms. It is shown that the evaluation process should be formulated as a multiple criteria decision-making problem under uncertainty. Three kinds of evaluating pattern are studied and put into action. Some experiments are reviewed and the main factors in the 
evaluating process are discussed. Needless to say, every pattern has its own strength and shortcomings so we can use them together to gain the synthesis conclusion of a kind of detection algorithms.

\section{ACKNOWLEDGE}

The authors would like to acknowledge the financial support by commonweal project of MOST.China -"Evaluating of Fire Detection Algorithm (2001DIB30124)" and sub item of national project '973'- "Multi-sensoring and Intelligent Identifying at Early Fire Stage (2001CB409608).”

\section{REFERENCES}

[1] Cleary, T., Grosshandler, W., and Chernovsky, A., "Smoke Detector Response to Nuisance Aerosols," Proceeding of AUBE 99', Duisburg, Germany, 1999, 3.

[2] Cleary, T., Donnelly, M., and Grosshandler, W., "The Fire Emulator/Detector Evaluator: Design, Operation, and Performance,” Proceedings of AUBE 01', Duisburg, Germany, 2001, 3.

[3] Gockel, F., "Fire Sensor Modeling and Simulation,” Proceedings of AUBE 01', Duisburg, Germany, 2001, 3.

[4] Li, J., Dong, W., Zhi-bin, M., "Evaluating Fire Detectors Using Fuzzy Analytic Hierarchy Process," Proceedings of AUBE'04 International Conference, Duisberg, Germany, 2004, 9, p. 307-311, Essen, Germany.

[5] Li, J., Dong, W., Zhi-bin, M., "Evaluation of Fire Detectors Performance," Proceedings of 2003 International Symposium on Fire Science and FireProtection Engineering, China Fire Protection Association, 2003, 10, p. 307311, Beijing, China.

[6] Annex L. Detection and Alarm Systems - Part 7: Point-type Smoke Detectors Using Scattered Light, Transmitted Light or Ionization, ISO/FDS 7240-7: 2003 (E).

[7] Rainer Siebel, "Test of Fire Detection Algorithm Using Artificially Generated Events," Proceedings of AUBE'04 International Conference, Duisberg, Germany, 2004, 9, p. 237-249, Essen, Germany. 
